\title{
Effect of erythropoietin on the expression of dynamin-related protein-1 in rat renal interstitial fibrosis
}

\author{
XIAN-FENG ZHAO ${ }^{1}$, YAN-HONG LIU ${ }^{2}$, ZI-MING HAN ${ }^{3}$ and $\mathrm{YU} \mathrm{XU}^{1}$ \\ ${ }^{1}$ Department of Cardiovascular Medicine, Henan Provincial People's Hospital, Zhengzhou, Henan 450000; \\ ${ }^{2}$ Department of Neonatology, Zhengzhou People's Hospital, Zhengzhou, Henan 450000; \\ ${ }^{3}$ Department of Pediatrics, The First Affiliated Hospital of Xinxiang Medical University, \\ Weihui, Henan 453100, P.R. China
}

Received March 21, 2014; Accepted November 7, 2014

DOI: $10.3892 / \mathrm{etm} .2015 .2419$

\begin{abstract}
This study aimed to observe the expression of dynamin-related protein-1 (Drp-1) in the renal interstitium in a rat model of renal interstitial fibrosis induced by unilateral ureteral obstruction (UUO). In addition, the renoprotective effect of erythropoietin in this model was investigated. A total of 81 rats were randomly assigned to sham surgery, UUO model and treatment groups. Following surgery, the rats in the treatment group were subcutaneously administered erythropoietin at a dose of 3,000 IU/kg once a week until the time of sacrifice. Rats in the sham surgery and UUO model groups were administered an identical volume of normal saline. In each group, nine rats were chosen randomly for sacrifice on days 7, 14 and 21 after surgery for histological examination of renal tissue. Renal tissue specimens were examined by hematoxylin and eosin and Masson's trichrome staining. Immunohistochemical analysis was performed to determine the expression of Drp-1 in the renal interstitium. Renal function damage, as evaluated by the measurement of serum creatinine $(\mathrm{Cr})$ and blood urea nitrogen (BUN) levels, was less severe in the treatment group compared with that in the model group at day $21 \quad(\mathrm{P}<0.01)$. Compared with the UUO model group, the renal interstitial injury score and fibrotic area of the treatment group were decreased markedly at the three time points $(\mathrm{P}<0.05)$. The expression level of Drp-1 in the treatment group was decreased markedly at the three time points compared with that in the model group $(\mathrm{P}<0.05)$. In conclusion, the expression of Drp-1 is increased in rat renal interstitial fibrosis, and erythropoietin may alleviate the degree of renal interstitial fibrosis by downregulating the expression of Drp-1.
\end{abstract}

Correspondence to: $\mathrm{Dr} \mathrm{Yu} \mathrm{Xu}$, Department of Cardiovascular Medicine, Henan Provincial People's Hospital, 33 Yellow River Road, Zhengzhou, Henan 450000, P.R. China

E-mail: yxxfen@126.com

Key words: dynamin-related protein-1, renal interstitial fibrosis, erythropoietin

\section{Introduction}

Renal interstitial fibrosis is the common pathway of a variety of kidney diseases that develop into end-stage renal failure (1). The process of renal interstitial fibrosis involves the loss of renal tubules and peritubular capillary endothelial cells, and the accumulation of inflammatory cells, interstitial myofibroblasts and extracellular matrix (2). A study has demonstrated that reductions in kidney function are more closely correlated with renal interstitial fibrosis levels than with glomerular sclerosis (3). Renal interstitial fibrosis is associated with a decline in renal function and is the most important prognostic marker $(1,4)$. Therefore, renal interstitial fibrosis is the focus of increasing attention and has become a popular research topic in international nephrology in recent years.

The pathogenesis of renal fibrosis is very complex. Studies have indicated that kidney diseases lead to renal tubular and interstitial cell apoptosis, affect renal remodeling and repair, and exacerbate renal interstitial fibrosis $(5,6)$. Furthermore, other studies have shown that apoptotic stimuli increase Drp-1 expression, induce mitochondrial division, cause changes in the expression and translocation of factors associated with apoptosis, and induce cell apoptosis $(7,8)$. Research suggests that more severe interstitial fibrosis is associated with increased cell apoptosis (9).

Studies suggest that erythropoietin (EPO) is a multifunctional cytokine superfamily member with a protective effect on multiple organs $(10,11)$. EPO has been demonstrated to exert a renoprotective effect in addition to a hematopoietic effect in acute and chronic kidney injury (12). It plays an important role in antioxidation, antiapoptosis and anti-inflammation in many models of kidney diseases $(13,14,15)$. A number of clinical studies have demonstrated that the early treatment of anemia in chronic kidney disease patients with EPO results in a slowing of the progressive decline in renal function (16-18).

In the present study, the renoprotective effect of EPO treatment was investigated in a unilateral ureteric obstruction (UUO) rat model of renal interstitial fibrosis. The hypothesis that EPO treatment may have a renoprotective effect that is mediated through modifying the expression of Drp-1 was also evaluated. 
Table I. Serum creatinine levels in all groups $(\mu \mathrm{mol} / \mathrm{l})$.

\begin{tabular}{lcccc}
\hline Groups & $\mathrm{n}$ & 7 days & 14 days & 21 days \\
\hline Sham surgery & 9 & $38.36 \pm 3.52$ & $43.05 \pm 5.27$ & $44.096 \pm 4.68$ \\
Model & 9 & $84.19 \pm 4.27^{\mathrm{a}}$ & $108.44 \pm 5.63^{\mathrm{a}, \mathrm{c}}$ & $157.43 \pm 5.73^{\mathrm{a}, \mathrm{c}}$ \\
Treatment & 9 & $59.62 \pm 2.78^{\mathrm{a}, \mathrm{b}}$ & $74.39 \pm 6.80^{\mathrm{a}-\mathrm{c}}$ & $113.32 \pm 6.21^{\mathrm{a}-\mathrm{c}}$ \\
F-value & & 146.293 & 214.086 & 207.425 \\
P-value & & $<0.05$ & $<0.05$ & $<0.05$ \\
\hline
\end{tabular}

Results are presented as the mean \pm standard deviation. ${ }^{\mathrm{a}} \mathrm{P}<0.05$ vs. the sham surgery group; ${ }^{\mathrm{b}} \mathrm{P}<0.05$ vs. the model group; ${ }^{\mathrm{c}} \mathrm{P}<0.05$ vs. the previous time point in the same group.

Table II. Blood urea nitrogen levels in all groups (mmol/l).

\begin{tabular}{lcccc}
\hline Groups & $\mathrm{n}$ & 7 days & 14 days & 21 days \\
\hline Sham surgery & 9 & $5.72 \pm 0.14$ & $6.03 \pm 0.35$ & $6.28 \pm 0.09$ \\
Model & 9 & $8.61 \pm 0.30^{\mathrm{a}}$ & $17.56 \pm 0.23^{\mathrm{a}, \mathrm{c}}$ & $24.56 \pm 1.42^{\mathrm{a}, \mathrm{c}}$ \\
Treatment & 9 & $6.49 \pm 1.28^{\mathrm{a}, \mathrm{b}}$ & $11.98 \pm 0.64^{\mathrm{a}-\mathrm{c}}$ & $16.84 \pm 0.47^{\mathrm{a}-\mathrm{c}}$ \\
F-value & & 79.264 & 113.489 & 161.731 \\
P-value & & $<0.05$ & $<0.05$ & $<0.05$ \\
\hline
\end{tabular}

Results are presented as the mean \pm standard deviation. ${ }^{a} \mathrm{P}<0.05$ vs. the sham surgery group; ${ }^{\mathrm{b}} \mathrm{P}<0.05$ vs. the model group; ${ }^{\mathrm{c}} \mathrm{P}<0.05$ vs. the previous time point in the same group.

\section{Materials and methods}

Animal care and model. This study was carried out in strict accordance with the recommendations in the Guide for the Care and Use of Laboratory Animals of the National Institutes of Health. The animal use protocol has been reviewed and approved by the Institutional Animal Care and Use Committee (IACUC) of Henan Provincial People's Hospital (Zhengzhou, China). The Sprague-Dawley rats, weighing 250-300 g, were obtained from Xinxiang Medical College Animal Center (Xinxiang, China). The rats were randomly divided into sham surgery, UUO model and treatment groups, with 27 rats in each group. The modeling method for the model and treatment groups was to ligate the left ureter and cut it. The modeling method for the sham surgery group was to free the ureter without ligation (19). The treatment group was administered EPO at a dose of $3,000 \mathrm{U} / \mathrm{kg}$ body weight by subcutaneous injection after surgery. The sham surgery and model groups were administered the same volume of saline by injection. Nine rats in each group were selected at random for sacrifice on each of days 7, 14 and 21 after surgery. Blood was collected from the heart at the time of sacrifice. Serum creatinine $(\mathrm{Cr})$ and blood urea nitrogen (BUN) levels in the blood were tested. Kidney specimens were fixed in $10 \%$ formalin solution and embedded in paraffin as 3- $\mu \mathrm{m}$ sections for hematoxylin and eosin (H\&E), Masson's trichrome and immunohistochemical staining.

Renal histology and immunohistochemistry. After H\&E and Masson's trichrome staining, the relative area of renal interstitial fibrosis was calculated using an IDA-2000 high-resolution digital image analysis system (Leica, Wetzlar, Germany). Ten non-overlapping visual fields were randomly selected in each slice in order to determine the renal interstitial fibrosis area as a percentage of the total interstitial area in the same visual field. The percentage of renal interstitial fibrosis in each slice was calculated as an average value. Renal interstitial injury was scored using a semi-quantitative scoring system. An average score was determined based on the score of each slice. The scoring system was as follows: 0 points, no lesions; 1 point, $<25 \%$ lesions; 2 points, $25-50 \%$ lesions; 3 points, $>50 \%$ lesions (20).

Immunohistochemical staining. The streptavidin biotin complex method was used to detect Drp-1 in the renal tissue. The staining procedure was conducted as described by the instructions provided with the Streptavidin Biotin Complex (SABC) kit (Wuhan Boster Biological Engineering Co., Ltd., Wuhan, China). Under a light microscope (SPZ-50 PFM; Carton Optical Industries, Ltd., Tokyo, Japan), histological sections were observed, and areas in which brown particles were deposited were positive staining areas. Under a high-power lens (magnification, x400), renal interstitial areas without glomeruli and vascular features were randomly selected in 10 non-overlapping visual fields. Images were collected and analyzed by the IDA-2000 high resolution digital image analysis system. The area with a gray level indicative of positive staining was calculated as a percentage of the total visual field, and an average value was determined (21). 
Table III. Renal tubulointerstitial damage score of all groups (\%).

\begin{tabular}{lcccc}
\hline Groups & $\mathrm{n}$ & 7 days & 14 days & 21 days \\
\hline Sham surgery & 9 & $0.49 \pm 0.03$ & $0.51 \pm 0.07$ & $0.52 \pm 0.04$ \\
Model & 9 & $3.41 \pm 0.18^{\mathrm{a}}$ & $5.07 \pm 0.26^{\mathrm{a}, \mathrm{c}}$ & $6.38 \pm 0.25^{\mathrm{a}, \mathrm{c}}$ \\
Treatment & 9 & $2.32 \pm 0.21^{\mathrm{a}, \mathrm{b}}$ & $3.94 \pm 0.15^{\mathrm{a}-\mathrm{c}}$ & $5.03 \pm 0.28^{\mathrm{a}-\mathrm{c}}$ \\
F-value & & 46.274 & 103.655 & 116.937 \\
P-value & & $<0.01$ & $<0.01$ & $<0.01$ \\
\hline
\end{tabular}

Results are presented as the mean \pm standard deviation. ${ }^{a} \mathrm{P}<0.05$ vs. the sham surgery group; ${ }^{\mathrm{b}} \mathrm{P}<0.05$ vs. the model group; ${ }^{\mathrm{c}} \mathrm{P}<0.05$ vs. the previous time point in the same group.

A

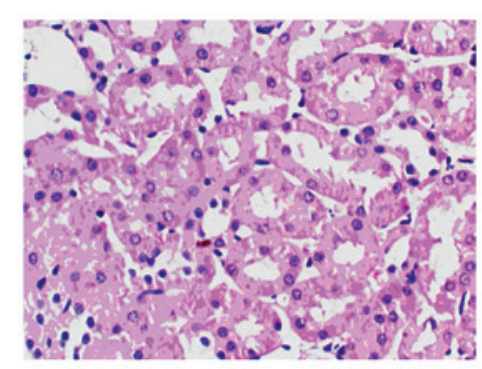

$\mathbf{D}$

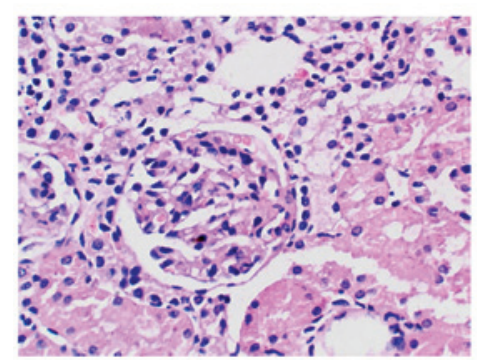

G

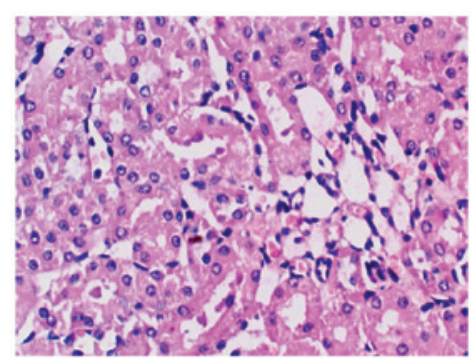

B

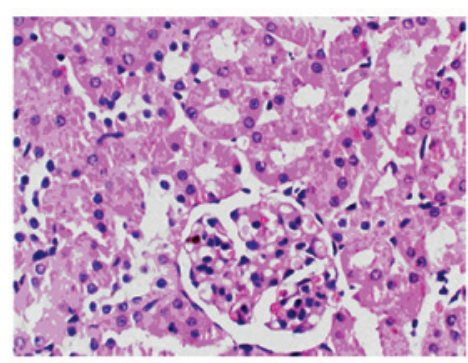

E

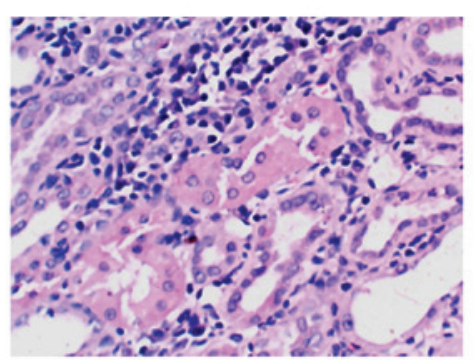

H

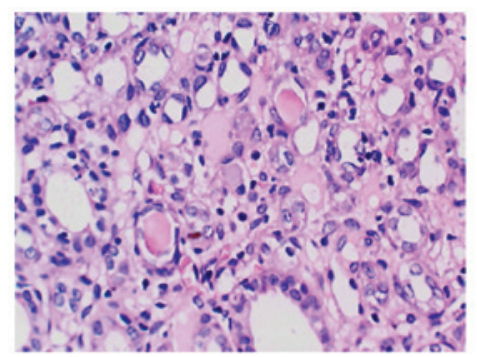

C

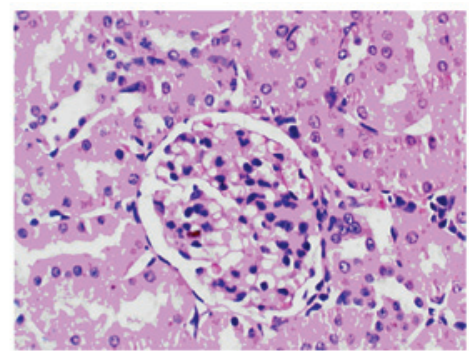

F

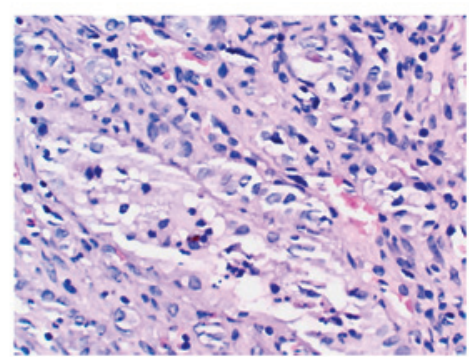

I

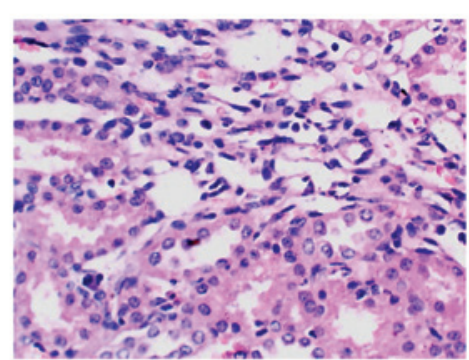

Figure 1. Hematoxylin and eosin staining for the assessment of interstitial fibrosis in mice following unilateral ureteral obstruction. Staining conducted (A) 7 , (B) 14 and (C) 21 days after surgery in the sham surgery group; (D) 7, (E) 14 and (F) 21 days after surgery in the model group; and (G) 7, (H) 14 and (I) 21 days after surgery in the treatment group (magnification, $\mathrm{x} 40$ ).

Statistical analysis. SPSS software, version 16.0 (SPSS, Inc., Chicago, IL, USA) was used. The data are expressed as the mean \pm standard deviation. The differences between groups were analyzed by single factor variance. Relevance judgments were analyzed by Pearson correlation. $\mathrm{P}<0.05$ was considered to indicate a statistically significant difference.

\section{Results}

Renal function. The serum $\mathrm{Cr}$ and BUN levels of the model and treatment groups were increased significantly compared with those in the sham surgery group at the same time point $(\mathrm{P}<0.05)$. However, the serum $\mathrm{Cr}$ and BUN levels of the treatment group were decreased significantly compared with those in the model group at each time point. The differences were statistically significant $(\mathrm{P}<0.05$; Tables I and II).

Kidney histology. H\&E and Masson's trichrome staining results showed that in the model group on day 7 after surgery, the renal interstitium was infiltrated by inflammatory cells, the cytoplasm of the renal tubular epithelial cells was loose with swelling and vacuolar degeneration, partial tubular dilation had occurred, the interstitium was widened, cells and extracellular matrix were increased in quantity, and fibrosis was visible in the cortex and the corticomedullary zone. On day 14 after surgery, the renal interstitium was markedly infiltrated by inflammatory cells, 
Table IV. Relative area of renal interstitial fibrosis in all groups (\%).

\begin{tabular}{lcccc}
\hline Groups & $\mathrm{n}$ & 7 days & 14 days & 21 days \\
\hline Sham surgery & 9 & $1.63 \pm 0.09$ & $1.72 \pm 0.11$ & $1.86 \pm 0.15$ \\
Model & 9 & $14.43 \pm 0.57^{\mathrm{a}}$ & $27.85 \pm 1.39^{\mathrm{a}, \mathrm{c}}$ & $53.16 \pm 2.45^{\mathrm{a}, \mathrm{c}}$ \\
Treatment & 9 & $9.77 \pm 0.62^{\mathrm{a}, \mathrm{b}}$ & $17.36 \pm 0.82^{\mathrm{a}-\mathrm{c}}$ & $31.29 \pm 1.94^{\mathrm{a}-\mathrm{c}}$ \\
F-value & & 85.033 & 126.572 & 187.836 \\
P-value & & $<0.01$ & $<0.01$ & $<0.01$ \\
& & & & \\
\hline
\end{tabular}

Results are presented as the mean \pm standard deviation. ${ }^{a} \mathrm{P}<0.05$ vs. the sham surgery group; ${ }^{\mathrm{b}} \mathrm{P}<0.05$ vs. the model group; ${ }^{\mathrm{c}} \mathrm{P}<0.05$ vs. the previous time point in the same group.

A

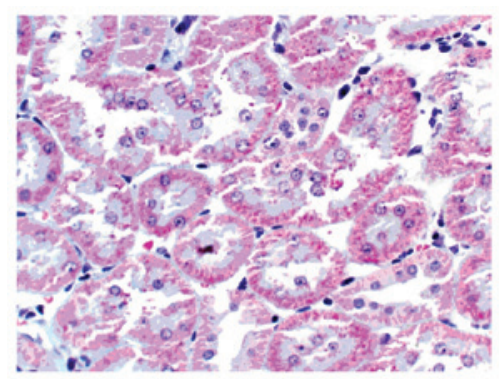

D

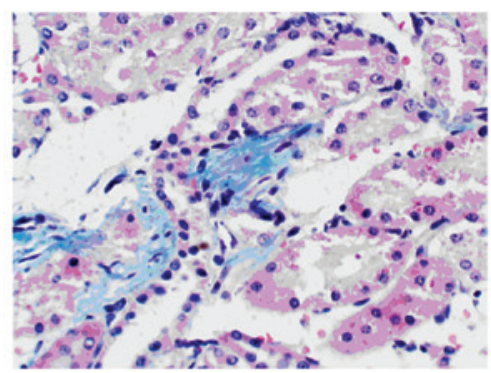

$\mathbf{G}$

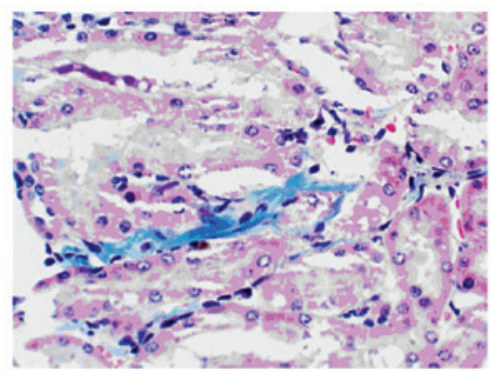

B

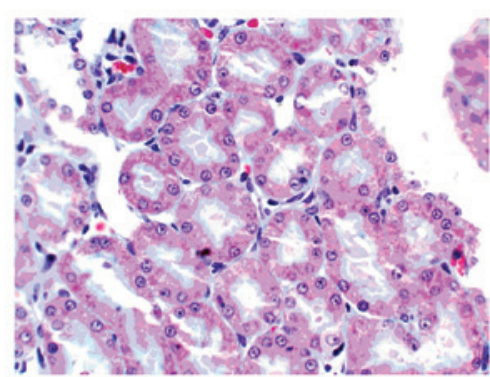

E

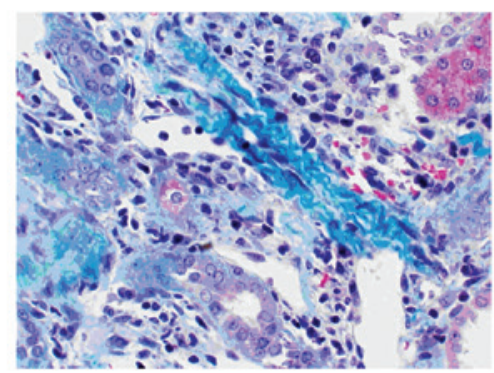

$\mathrm{H}$ 3

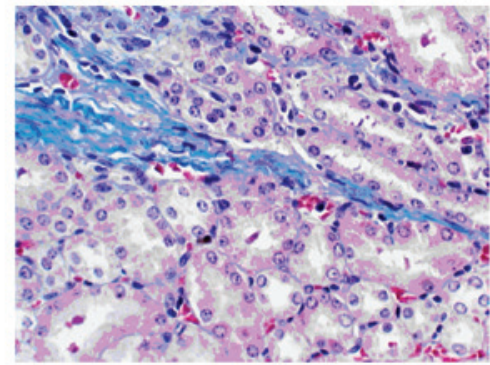

C

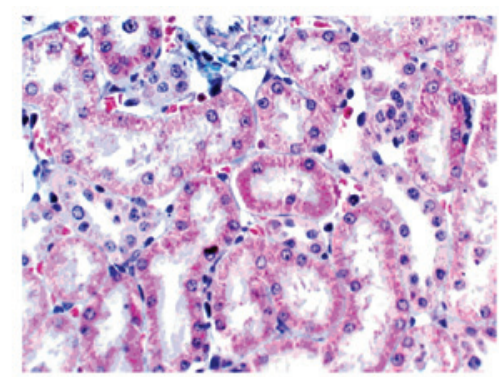

F

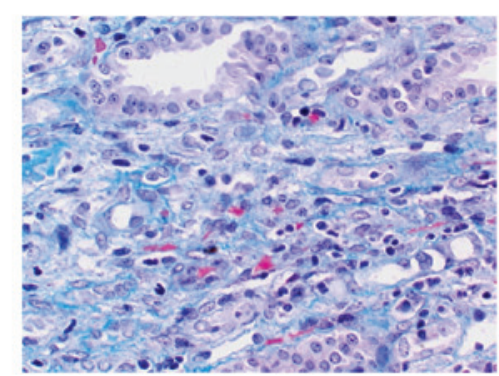

I

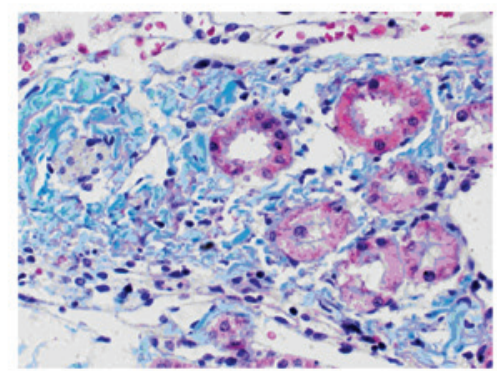

Figure 2. Masson's trichrome staining for the assessment of interstitial fibrosis in mice following unilateral ureteral obstruction. Staining conducted (A) 7 , (B) 14 and (C) 21 days after surgery in the sham surgery group; (D) 7, (E) 14 and (F) 21 days after surgery in the model group; and (G) 7, (H) 14 and (I) 21 days after surgery in the treatment group (magnification, $\mathrm{x} 40$ ).

tubular dilatation was evident, partial shedding and atrophy of tubular epithelial cells was visible, the interstitium was widened significantly, and the cortex and corticomedullary zone were clearly fibrotic. On day 21 after surgery, the renal interstitium was diffusely infiltrated by inflammatory cells, the renal tubular structures were seriously damaged, tubular dilation, deformation and atrophy were severe, tubulointerstitial broadening was more evident, and fibrous hyperplasia was observed. The renal tissue of the sham surgery group showed none of these changes. The treatment group at each time point was compared with the model group. In the treatment group, renal interstitial infiltration by inflammatory cells was reduced, tubular dilation and atrophy were attenuated significantly, the area of renal interstitial fibrosis was reduced and the renal interstitial injury score was significantly decreased $(\mathrm{P}<0.05)$, although it remained higher than that in the sham surgery group $(\mathrm{P}<0.01)$. The renal tubular interstitial damage scores are shown in Table III and Fig. 1, and the relative area of renal interstitial fibrosis is shown in Table IV and Fig. 2.

Immunohistochemistry. Drp-1 in the sham surgery group at each time point was expressed at low levels in renal 
Table V. Relative expression area of Drp-1 in renal tissue of the three groups at different time points $(\%)$.

\begin{tabular}{lcccc}
\hline Groups & $\mathrm{n}$ & 7 days & 14 days & 21 days \\
\hline Sham surgery & 9 & $0.88 \pm 0.09$ & $1.05 \pm 0.14$ & $1.06 \pm 0.17$ \\
Model & 9 & $9.92 \pm 0.37^{\mathrm{a}}$ & $16.42 \pm 0.58^{\mathrm{ac}}$ & $25.74 \pm 0.19^{\mathrm{a}, \mathrm{c}}$ \\
Treatment & 9 & $6.05 \pm 0.21^{\mathrm{a}, \mathrm{b}}$ & $11.28 \pm 0.13^{\mathrm{a}-\mathrm{c}}$ & $18.63 \pm 0.22^{\mathrm{a}-\mathrm{c}}$ \\
F-value & & 153.628 & 428.371 & 507.845 \\
P-value & & $<0.05$ & $<0.05$ & $<0.05$ \\
\hline
\end{tabular}

Results are presented as the mean \pm standard deviation. ${ }^{a} \mathrm{P}<0.05$ vs. the sham surgery group; ${ }^{\mathrm{b}} \mathrm{P}<0.05$ vs. the model group; ${ }^{\mathrm{c}} \mathrm{P}<0.05$ vs. the previous time point in the same group.

$\mathbf{A}$

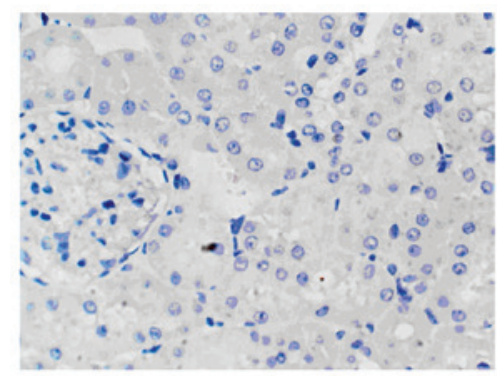

D

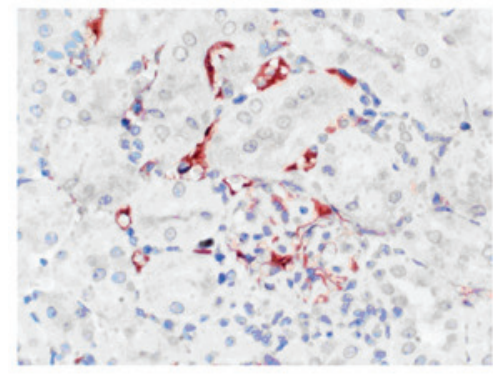

G

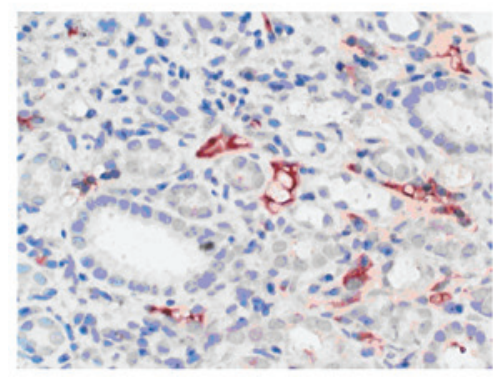

B

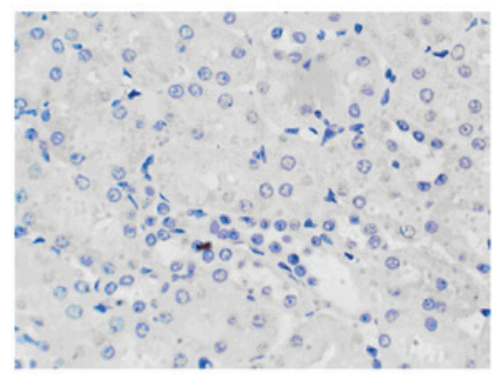

E

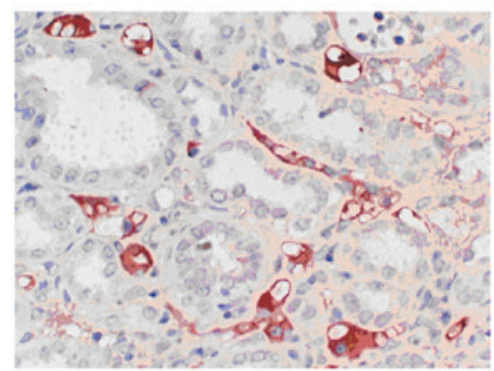

H

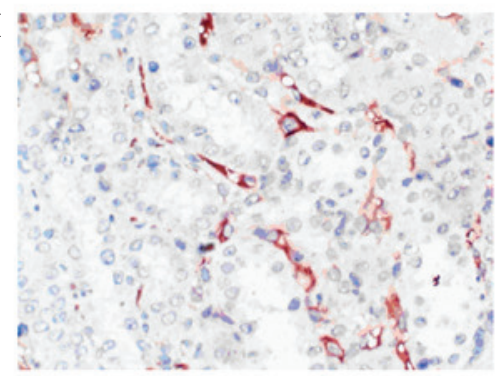

C

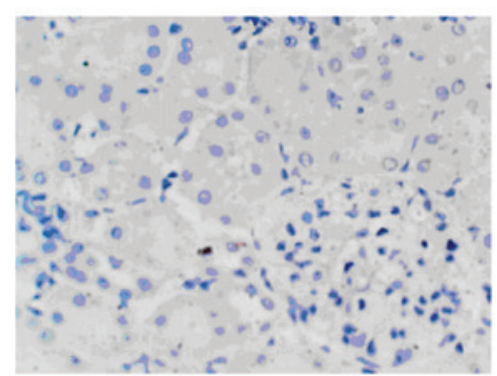

F

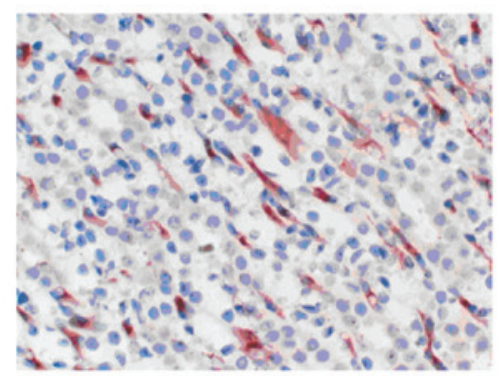

I

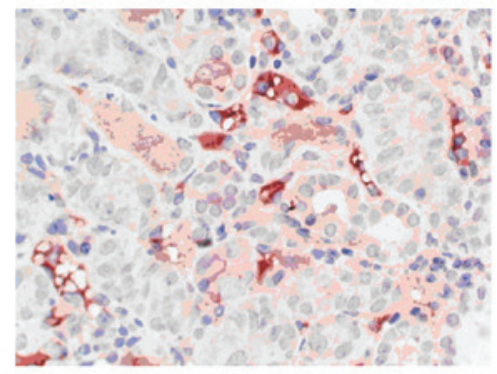

Figure 3. Representative images of kidney sections stained for Drp-1 in mice following unilateral ureteral obstruction. Staining conducted (A) 7, (B) 14 and (C) 21 days after surgery in the sham surgery group; (D) 7, (E) 14 and (F) 21 days after surgery in the model group; and (G) 7, (H) 14 and (I) 21 days after surgery in the treatment group (magnification, $\mathrm{x} 400$ ). Drp-1, dynamin-related protein-1.

interstitial and tubular epithelial cells. In the model group, the expression of Drp-1 was located in the cell cytoplasm of the renal tubular epithelial cells and interstitium, and the expression levels gradually increased with the prolongation of UUO. In the treatment and model groups, the sites of expression were essentially the same, but the scope and intensity were decreased in the treatment group. Parallel comparison showed that Drp-1 expression in the model and treatment groups at each time point was significantly increased compared with that in the sham surgery group $(\mathrm{P}<0.05)$, and that Drp-1 expression in the treatment group was reduced compared with that in the model group. The difference was statistically significant $(\mathrm{P}<0.05$; Table $\mathrm{V}$ and Fig. 3).

Correlation analysis. By analyzing the area of positive Drp-1 expression, renal tubulointerstitial injury, and relative area of renal interstitial fibrosis of the model and treatment groups, it was identified that the positively stained area of Drp-1, renal tubulointerstitial injury and relative area of renal interstitial fibrosis were positively correlated $(r=0.923$, 0.895). 


\section{Discussion}

Renal interstitial fibrosis is the common pathway for a variety of chronic kidney disease as they develop to end-stage renal failure. The pathological features include renal interstitial fibroblast proliferation and the excessive accumulation of extracellular matrix (22). There is evidence that, in renal interstitial fibrosis, there is a close relationship between the apoptosis of renal tubular epithelial cells and tubular atrophy; the increase in the apoptosis index is one of the causes (23) of extracellular matrix formation. A study suggests that more severe interstitial fibrosis is associated with increased cell apoptosis, with a positive correlation existing between the degree of interstitial fibrosis and the apoptotic cell number (24). One of the main processes involved in apoptosis is mitochondrial fracture. Drp-1 in mammalian cells is an important executive molecule for mitochondrial fission. Drp-1 is mainly located in the cytoplasm as a polymer. It can be translocated to the outer mitochondrial membrane and accumulated into the potential sites of mitochondrial division when it is recruited by the molecules of the mitochondrial outer membrane. Multiple Drp-1 molecules surrounding the mitochondria ultimately lead to the breakdown of the mitochondria and apoptosis (25). A previous study found that the overexpression of Drp-1 may lead to an acceleration of mitochondrial fission, resulting in the production of a large number of mitochondrial fragments (26). The inhibition of endogenous Drp-1 by gene knockout can inhibit mitochondrial fission (27). A study of the apoptosis of renal tubular epithelial cells induced by ATP depletion found that the activation of Drp-1 leads to the fragmentation of mitochondria, resulting in the release of intermembrane pro-apoptotic proteins, and induction of the apoptosis of cells (28).

Erythropoietin is bone marrow stimulation factor, synthesized by the kidneys and liver, and a multifunctional member of the cytokine super family (29). A previous study found that EPO can ameliorate anemia, inhibit inflammatory reactions, promote the growth of blood vessels, inhibit apoptosis, and reduce the expression of cytokines and inflammatory factors, thereby protecting organs from damage (30). In recent years, a large number of animal experiments have shown that EPO can delay the progress of chronic kidney disease and improve the degree of interstitial fibrosis, and has a certain renal protective effect (31-33). A study conducted by Nakazawa et al demonstrated that EPO is able to inhibit renal tubule cell apoptosis, thereby reducing the degree of renal interstitial fibrosis (34).

In the present study, a rat model of UUO was established to study the expression of Drp-1 in renal interstitial fibrosis and the ability of erythropoietin to protect against it. The results showed that 7, 14, and 21 days after surgery, in the treatment group at each time point compared with the model group, the renal function was improved significantly and infiltration of the renal interstitium by inflammatory cells was reduced. In addition, tubular dilatation and atrophy, the relative area of renal interstitial fibrosis, and the renal interstitial injury score were markedly decreased. Immunohistochemical analysis showed that in the model and treatment groups at each time point, Drp-1 expression levels were significantly increased compared with those in the sham surgery group $(\mathrm{P}<0.05)$. The Drp-1 expression levels in the kidneys of rats in the treatment group were decreased significantly compared with those in the model group at the same time point. The results of correlation analysis showed that the expression of Drp-1 in renal tissue, the renal interstitial injury score and relative area of renal interstitial fibrosis were positively correlated. The results indicate that erythropoietin may reduce apoptosis and decrease renal interstitial fibrosis by inhibiting the expression of Drp-1 in renal tissue.

In summary, the overexpression of Drp-1 is potentially one of the mechanisms underlying the development of renal interstitial fibrosis. Erythropoietin may delay the development of renal interstitial fibrosis and protect the kidney by inhibiting the expression of Drp-1 in the renal tissue of rats subjected to UUO. However, the mechanism by which erythropoietin inhibits the expression of Drp-1 in the renal tissue in this model requires further study.

\section{References}

1. Nangaku M: Mechanisms of tubulointerstitial injury in the kidney: final common pathways to end-stage renal failure. Intern Med 43: 9-17, 2004

2. Campanholle G, Ligresti G, Gharib SA and Duffield JS: Cellular mechanisms of tissue fibrosis 3. Novel mechanisms of kidney fibrosis. Am J Physiol Cell Physiol 304: C591-C603, 2013.

3. Eddy AA: Molecular basis of renal fibrosis. Pediatric Nephrol 15: 290-301, 2000.

4. Ucero AC, Benito-Martin A, Izquierdo MC, et al: Unilateral ureteral obstruction: beyond obstruction. Int Urol Nephrol 46: 765-776, 2014.

5. Strutz F: Pathogenesis of tubulointerstitial fibrosis in chronic allograft dysfunction. Clin Transplant 23 (Suppl 21) : 26-32, 2009.

6. Ucero AC, Gonçalves S, Benito-Martin A, et al: Obstructive renal injury: from fluid mechanics to molecular cell biology. Open Access J Urol 2: 41-55, 2010.

7. Otera $\mathrm{H}$ and Mihara K: Molecular mechanisms and physiologic functions of mitochondrial dynamics. J Biochem 149: 241-251, 2011.

8. Ucero AC, Benito-Martin A, Fuentes-Calvo I, et al: TNF-related weak inducer of apoptosis (TWEAK) promotes kidney fibrosis and Ras-dependent proliferation of cultured renal fibroblast. Biochim Biophys Acta 1832: 1744-1755, 2013.

9. Izquierdo MC, Sanz AB, Mezzano S, et al: TWEAK (tumor necrosis factor-like weak inducer of apoptosis) activates CXCL16 expression during renal tubulointerstitial inflammation. Kidney Int 81: 1098-1107, 2012.

10. Brines $M$ and Cerami A: Discovering erythropoietin's extra-hematopoietic functions: biology and clinical promise. Kidney Int 70: 246-250, 2006.

11. Baker JE: Enythropoietin mimics ischemic preconditioning. Vascul Pharmacol 42: 233-241, 2005.

12. Chang YK, Choi DE, Na KR, et al: Erythropoietin attenuates renal injury in an experimental model of rat unilateral ureteral obstruction via anti-inflammatory and anti-apoptotic effects. J Urol 181: 1434-1443, 2009.

13. Cassis P, Gallon L, Benigni A, et al: Erythropoietin, but not the correction of anemia alone, protects from chronic kidney allograft injury. Kidney Int 81: 903-918, 2012.

14. Yu XQ, Wu LL, Huang XR, et al: Osteopontin expression in progressive renal injury in remnant kidney:rule of angiotension II. Kidney Int 58: 1469-1480, 2000.

15. Li C, Chen Y, Hong MY: Erythropoietin protective effect of renal perfusion by pretreatment on acute global ischemia in rats. Zhongguo Bingli Shengli Zazhi 20: 2336-2338, 2004.

16. Srisawat N, Manotham K, Eiam-Ong S, Katavetin P, Praditpornsilpa K and Eiam-Ong S: Erythropoietin and its non-erythropoietic derivative: do they ameliorate renal tubulointerstitial injury in ureteral obstruction? Int J Urol 15: 1011-1017, 2008.

17. Sharples EJ, Patel N, Brown P, et al: Erythropoietin protects the kidney against the injury and dysfunction caused by ischemiareperfusion. J Am Soc Nephrol 15: 2115-2124, 2004. 
18. Ates E, Yalcin AU, et al: Protective effect of erythropoietin on renal ischemia and reperfusion injury. ANZ J Surg 75: 1100-1105, 2005.

19. Chevalier RL, Forbes MS and Thornhill BA: Ureteral obstruction as a model of renal interstitial fibrosis and obstructive nephropathy. Kidney Int 75: 1145-1152, 2009.

20. Pang M, Kothapally J, Mao H, et al: Inhibition of histone deacetylase activity attenuates renal fibroblast activation and interstitial fibrosis in obstructive nephropathy. Am J Physiol Renal Physiol 297: F996-F1005, 2009.

21. Cregger M, Berger AJ and Rimm DL: Immunohistochemistry and quantitative analysis of protein expression. Arch Pathol Lab Med 130: 1026-1030, 2006

22. Hewitson TD, Ho WY and Samuel CS: Antifibrotic properties of relaxin: in vivo mechanism of action in experimental renal tubulointerstitial fibrosis. Endocrinol 151: 4938-4948, 2010.

23. Yang T, Vesey DA, Johnson DW, Wei MQ and Gobe GC: Apoptosis of tubulointerstitial chronic inflammatory cells in progressive renal fibrosis after cancer therapies. Transl Res 150: 40-50, 2007.

24. Izquierdo MC, Sanz AB, Mezzano S, et al: TWEAK (tumor necrosis factor-like weak inducer of apoptosis) activates CXCL16 expression during renal tubulointerstitial inflammation. Kidney Int 81: 1098-1107, 2012

25. Figueroa-Romero C, Iñiguez-Lluhí JA, Stadler J, et al: SUMOylation of the mitochondrial fission protein Drpl occurs at multiple nonconsensus sites within the B domain and is linked to its activity cycle. FASEB J 23: 3917-3927, 2009.

26. Brooks C, Cho SG, et al: Fragmented mitochondria are sensitized to Bax insertion and activation during apoptosis. Am J Physiol Cell Physiol 300: C447-C455, 2011.
27. Wang H, Lim PJ, Karbowski M and Monteiro MJ. Effects of overexpression of huntingtin proteins on mitochondrial integrity. Hum Mol Genet 18: 737-752, 2009.

28. Brooks C, Cho SG, Wang CY, Yang T and Dong Z: Fragmented mitochondria are sensitized to Bax insertion and activation during apoptosis. Am J Physiol Cell Physiol 300: C447-C455, 2011.

29. Ghezzi P and Brines M: Erythropoietin as an antiapoptotic, tissue-protective cytokine. Cell Death Differ 11 (Suppl 1): S37-S44, 2004.

30. Ergur BU, Kiray M, Pekcetin C, et al: Protective effect of erythropoietin pretreatment in testicular ischemia-reperfusion injury in rats. J Pediatr Surg 43: 722-728, 2008.

31. Kitamura H, Isaka Y, Takabatake Y, et al: Nonethropoietic derivative of erythropoietin protect against tubulointerstitial injury in a unilateral ureteral obstruction model. Nephrol Dial Transplant 23: 1521-1528, 2008.

32. Kuriyams S, Tomonari H, Tokudome G, et al: Association of angiotensinogen gene polymorphism with erythropoietininduced hypertension: a preliminary report. Hypertens Res 24: 501-505, 2001

33. Jungers P, Choukroun G, Oualim Z, Robino C, Nguyen AT and Man NK: Beneficial influence of recombinant human erythropoietin therapy on the rate of progression of chronic renal failure in predialysis patients. Nephrol Dial Transplant 16: 307-312, 2001

34. Nakazawa Y, Nishino T, Obata K, et al: Recombinant human erythropoietin attenuates renal tubulointerstitial injury in murine adriamycin-induced nephropathy. J Nephrol 26: $527-533,2013$ 